\title{
Linear analysis of the vectorial network model in the presence of leaders ${ }^{\text {th }}$
}

\author{
Violet Mwaffo, Maurizio Porfiri*
}

Department of Mechanical and Aerospace Engineering, New York University Polytechnic School of Engineering, Brooklyn, NY, 11201 USA

\begin{abstract}
In this work, we study the collective behavior of a multi-agent system of stochastically interacting leaders and followers. In this time-varying network, the nodes update their states through a noisy interaction with a randomly selected subset of neighbors, including leaders whose average orientation is not updated in time. By linearizing the system dynamics in the vicinity of the leaders' common trajectory, we establish a toolbox of closed-form expressions that aid in the understanding of the influence of noise, number of connected neighbors, network size, and proportion of leaders on the group coordination.
\end{abstract}

Keywords: Consensus, leader-follower, networks, polarization, stochastic stability, synchronization

\section{Introduction}

Collective behavior is a common phenomenon in several biological groups, such as bird flocks, fish shoals, and insect swarms $[1,2,3]$. This phenomenon is often the result of local interactions among the group's individuals $[4,5]$ and is manifested in the form of highly coordinated actions that have inspired the solution of several engineering problems in the areas of synchronization, including neural networks $[6,7]$ and communications $[8,9]$. Biological groups can be modeled as a network of coupled dynamical systems, in which the nodes correspond to the individuals and the edges represent the interactions among them [10]. Studies on biological groups indicate that knowledge about the environment may significantly vary among individuals, whereby some may have access to information that is not available to others [4]. Often, a few informed individuals may act as group leaders by influencing the uninformed conspecifics, and thus modulating the overall collective response [11]. Upon modeling the group as a network of dynamical systems, the emergence of leader-follower relationships is analogous to pinning control $[12,13]$.

An effective network model used to describe collective behavior is the vectorial network model (VNM), introduced in Ref. [14] as a simplification of the classical Vicsek model $[15,16]$, which has found extensive applications in engineering and biology to describe the emergent behavior of social animals and multi-vehicle teams [17]. The VNM consists of $N$ interacting agents represented by twodimensional vectors expressed as complex numbers $v_{1}=$

This work was supported by the National Science Foundation under Grant \# CMMI-0745753 and GK-12 Fellows Grant \# DGE0741714

${ }^{*}$ Corresponding author. Tel.: +1 718260 3681; fax: +1 718260 3532 .

Email address: mporfiri@nyu.edu (Maurizio Porfiri)
$\mathrm{e}^{\mathrm{I} \theta_{1}}, \ldots, v_{N}=\mathrm{e}^{\mathrm{I} \theta_{N}}$, where $\theta_{i}, i=1, \ldots, N$ are the angles defining the orientation of each agent, and I is the imaginary unit. In a discrete-time setting, the agents interact with a subset of $K$ randomly selected agents, from which they update their orientation. Thus, each agent determines its orientation at the next time step from the average of the two-dimensional vectors corresponding to its neighbors, subject to noise. The random selection of neighbors in this model is executed at each time step, and prevents the coupling between the orientation of the agents and their local density as in the Vicsek model $[15,16,18,19]$. Similar metric-free models have been recently presented in Refs. [20, 21].

To model, for the first time, the presence of informed individuals in the VNM, we assume that a proportion $\phi$ of the agents are leaders. Without loss of generality, we hypothesize that the nodes $i=1, \ldots, N_{f}=(1-\phi) N$ are followers that update their orientation based on the state of $K$ neighbors, and the $N_{l}=\phi N$ remaining nodes, $i=$ $N_{f}+1, \ldots, N$, are leaders that share a common reference state $\theta_{0}$. With respect to biological groups, such common orientation may be associated with a migration route or a food source [4]. At each time $k \in \mathbb{Z}^{+}$, the node $i, i=$ $1, \ldots, N$, computes the following average vector:

$$
U_{i}(k)= \begin{cases}\frac{1}{K} \sum_{j=1}^{K} v_{i_{j}}(k), & \text { if } i \text { is a follower, } \\ v_{i}, & \text { if } i \text { is a leader, }\end{cases}
$$

where $i_{1}, \ldots, i_{K}$ are the agents connected to node $i$; this set can also include node $i$.

The orientations of the agents at any time $k \in \mathbb{Z}^{+}$can be written in a compact form as $\theta(k)=\left[\theta^{f}(k)^{\mathrm{T}}, \theta^{l}(k)^{\mathrm{T}}\right]^{\mathrm{T}}$, where $\theta^{f}(k)=\left[\theta_{1}(k), \ldots, \theta_{N_{f}}(k)\right]^{\mathrm{T}}$ is the state of the followers and $\theta^{l}(k)=\left[\theta_{N_{f}+1}(k), \ldots, \theta_{N}(k)\right]^{\mathrm{T}}$ is the state of 
the leaders. Agents are also subject to intrinsic noise, and the dynamics of the system is given by

$$
\theta_{i}(k+1)= \begin{cases}\arg \left\{U_{i}(k)\right\}+\eta \zeta_{i}(k), & \text { if } i \text { is a follower, } \\ \theta_{0}+\eta \zeta_{i}(k), & \text { if } i \text { is a leader }\end{cases}
$$

where $0 \leq \eta \leq 1$ quantifies the noise intensity, $\arg (\cdot)$ is the argument of a complex number, $\zeta_{1}(k), \ldots, \zeta_{N}(k)$ are independent and identically distributed (i.i.d.) random variables with common uniform random variable taking values in $[-\pi, \pi]$.

In the absence of leaders, studies on the VNM have investigated the effects of group size $N$, number of connected neighbors $K$, and noise intensity $\eta$ on the system coordination $[14,18,22]$. Specifically, in the thermodynamic limit $N \rightarrow \infty$, it has been shown that the system exhibits a continuous order-disorder phase transition $[14,18]$. In other words, the vectors synchronize toward a common orientation for $\eta=0$, and the degree of synchronization smoothly decreases as noise increases, until a phase transition to a completely disordered state is observed. The critical noise at which such phase transition occurs is controlled by $K$, and a closed-form solution for $K \rightarrow \infty$ has been presented in Ref. [18]. To investigate the VNM beyond the thermodynamic limit and thus offer valuable insight into the coordination of biological groups, an alternative methodology has been proposed in Ref. [22]. Therein, the system dynamics is linearized for $\eta \ll 1$, resulting in a linear stochastic consensus problem $[23,24,25,26,27,28,29,30,31,32]$. By adapting the algebraic analysis proposed for mean square consensus in Refs. [33, 34, 35], a toolbox of closed-form results for the system dynamics are established. Numerical findings from independent simulations suggest that such analytical expressions accurately reproduce the system response for noise levels in the range $\eta \lesssim 0.5$.

In this work, we extend the methodology presented in Ref. [22] to investigate the effect of group leaders on the coordination of the VNM. We specifically focus on the linear stochastic dynamics stemming from (1) and (2) for $\eta \ll 1$ and on small variations of the orientation of the followers with respect to the leaders. Hence, we define the disagreement between the angle of an agent $i$ and the reference angle $\theta_{0}$ by $\varepsilon_{i}(k)=\theta_{i}(k)-\theta_{0}$. For small variations $\varepsilon_{1}(k), \ldots, \varepsilon_{N}(k)$, we linearize the model in equation (2) to obtain the linear stochastic dynamics of the disagreement, that is,

$$
\varepsilon_{i}(k+1)= \begin{cases}\frac{1}{K} \sum_{j=1}^{K} \varepsilon_{i_{j}}(k)+\eta \zeta_{i}(k), & \text { if } i \text { is a follower } \\ \eta \zeta_{i}(k), & \text { if } i \text { is a leader. }\end{cases}
$$

For convenience, we write the disagreement vector as $\varepsilon(k)=\left[\varepsilon^{f}(k)^{\mathrm{T}}, \varepsilon^{l}(k)^{\mathrm{T}}\right]^{\mathrm{T}}$ with $\varepsilon^{f}(k)=\left[\varepsilon_{1}(k), \ldots, \varepsilon_{N_{f}}(k)\right]^{\mathrm{T}}$ $\in \mathbb{R}^{N_{f}}$ and $\varepsilon^{l}(k)=\left[\varepsilon_{N_{f}+1}(k), \ldots, \varepsilon_{N}(k)\right]^{\mathrm{T}} \in \mathbb{R}^{N_{l}} ;$ and, similarly, we write the intrinsic noise vector as $\zeta(k)=$ $\left[\zeta^{f}(k)^{\mathrm{T}}, \zeta^{l}(k)^{\mathrm{T}}\right]^{\mathrm{T}}$, where $\zeta^{f}(k)=\left[\zeta_{1}(k), \ldots, \zeta_{N_{f}}(k)\right]^{\mathrm{T}} \in$ $\mathbb{R}^{N_{f}}$ and $\zeta^{l}(k)=\left[\zeta_{N_{f}+1}(k), \ldots, \zeta_{N}(k)\right]^{\mathrm{T}} \in \mathbb{R}^{N_{l}}$, respectively. Using this compact notation, the linear stochastic system (3) can be rewritten as

$$
\varepsilon(k+1)=\mathcal{C}(k) \varepsilon(k)+\eta \zeta(k),
$$

where $\mathcal{C}(k)$ are the state matrices. Similar to Ref. [22], $\mathcal{C}(k)$ are i.i.d. matrices with common random variable $\mathcal{C}$, which is defined so that its first $N_{f}$ rows are i.i.d. vectors with $K$ randomly selected entries taking value $\frac{1}{K}$ while all other entries equal 0. Similar to most of the classical studies on stochastic consensus protocols [23, 25, 26], this matrix is non-negative; however it is generally nonsymmetric and, most importantly, its entries are not independent random variables. We further note that the problem shares similarities with Ref. [35], which addresses leader-follower consensus in numerosity-constrained networks. However, differently than Ref. [35], we consider a noisy consensus protocol which stems from the linearization of a nonlinear model. The resulting state matrix differs from the numerosity-constrained network model in Ref. [35], whereby each agent is not required to utilize its past orientation during the updating process. As a result, the diagonal entries of the state matrix may vary in time, and the agents interact with $K$ or $K-1$ others.

In what follows, we refer by $I_{N}$ the $N \times N$ identity matrix; the $N \times 1$ vector of all ones is referred by $1_{N}$; the $N \times 1$ vector of all zeros is $0_{N}$ and $0_{N \times M}$ is the $N \times M$ matrix of all zeros; the Kronecker product of two matrices $A$ and $B$ is $A \otimes B$; the operation $\operatorname{vec}(\cdot)$ is the vectorization of a matrix by stacking its columns; the matrix transposition is referred by a superscript $\mathrm{T}$; and $\mathbf{E}[\cdot]$ refers to the expected value of a random variable.

\section{Asymptotic behavior}

\subsection{Mean square analysis}

Following [33, 34, 35], we consider the second moment matrix, sometimes referred to as autocorrelation, $\Xi(k)=$ $\mathbf{E}\left[\varepsilon(k) \varepsilon(k)^{\mathrm{T}}\right]$ to investigate the mean square behavior of the stochastic system (3). Notably, the trace of such a matrix measures the mean square deviation, that is, $\delta(k)=$ $\mathbf{E}\left[\|\varepsilon(k)\|^{2}\right]=\operatorname{vec}\left(I_{N}\right)^{\mathrm{T}} \operatorname{vec}(\Xi(k))$.

Following the decomposition in Ref. [35], the leaders do not update their state on the basis of their neighbors, and the linearized model is reduced to contain only the followers' states. Hence, we write the first $N_{f}$ rows of $\mathcal{C}(k)$ as the augmented matrix $\left[\mathcal{C}^{f f}(k) \mid \mathcal{C}^{f l}(k)\right]$, where $\mathcal{C}^{f f}(k) \in$ $\mathbb{R}^{N_{f} \times N_{f}}$ and $\mathcal{C}^{f l}(k) \in \mathbb{R}^{N_{f} \times N_{l}}$ are i.i.d. matrices with common random variables $\mathcal{C}^{f f}$ and $\mathcal{C}^{f l}$, respectively. Thus, we have

$$
\mathcal{C}=\left[\begin{array}{c|c}
\mathcal{C}^{f f} & \mathcal{C}^{f l} \\
\hline 0_{N_{l} \times N_{f}} & 0_{N_{l} \times N_{l}}
\end{array}\right] .
$$


Using this decomposition, the dynamics of the followers' disagreement become

$$
\varepsilon^{f}(k+1)=\mathcal{C}^{f f}(k) \varepsilon^{f}(k)+\eta\left(\zeta^{f}(k)+\mathcal{C}^{f l}(k) \zeta^{l}(k-1)\right),
$$

while the dynamics of the leaders' disagreement are simply

$$
\varepsilon^{l}(k+1)=\eta \zeta^{l}(k) .
$$

By recalling the independence of $\zeta_{1}(k), \ldots, \zeta_{N}(k)$, the second moment matrix $\Xi(k)$ reduces to the following block diagonal matrix:

$$
\Xi(k)=\left[\begin{array}{c|c}
\Xi^{f}(k) & 0_{N_{f} \times N_{l}} \\
\hline 0_{N_{l} \times N_{f}} & \Xi^{l}(k)
\end{array}\right],
$$

where $\Xi^{f}(k)=\mathbf{E}\left[\varepsilon^{f}(k) \varepsilon^{f}(k)^{\mathrm{T}}\right]$ and $\Xi^{l}(k)=\mathbf{E}\left[\varepsilon^{l}(k) \varepsilon^{l}(k)^{\mathrm{T}}\right]$ are the second moment matrices of the followers' and the leaders' states, respectively.

Considering that the noise components are uniform i.i.d. random variables in $[-\pi, \pi]$, the noise covariance matrix is $\mathbf{E}\left[\zeta \zeta^{\mathrm{T}}\right]=\frac{\pi^{2}}{3} I_{N}$. By replacing in the previous result $\zeta$ with $\zeta^{l}$, and using equation $(6 \mathrm{~b})$, the leaders' second moment matrix is

$$
\mathbf{E}\left[\varepsilon^{l}(k) \varepsilon^{l}(k)^{\mathrm{T}}\right]=\eta^{2} \frac{\pi^{2}}{3} I_{N_{l}} .
$$

We compute the evolution of the second moment matrix for the followers by iterating (6a) from time 0 to time $k$, using a well-known property of the Kronecker algebra [36], and recalling that $\zeta$ and $\mathcal{C}$ are independent random variables with $\mathbf{E}[\zeta]=0$. Thus, we find

$$
\begin{aligned}
& \operatorname{vec}\left(\Xi^{f}(k+1)\right)=G_{1}^{k+1} \operatorname{vec}\left(\varepsilon(0) \varepsilon(0)^{\mathrm{T}}\right)+ \\
& \eta^{2}\left(\sum_{s=0}^{k}\left(G_{1}\right)^{s}\right)\left(G_{2} \operatorname{vec}\left(\mathbf{E}\left[\zeta^{l} \zeta^{l^{\mathrm{T}}}\right]\right)+\operatorname{vec}\left(\mathbf{E}\left[\zeta^{f} \zeta^{\left.f^{\mathrm{T}}\right]}\right]\right),\right.
\end{aligned}
$$

where $G_{1}=\mathbf{E}\left[\mathcal{C}^{f f} \otimes \mathcal{C}^{f f}\right] \in \mathbb{R}^{N_{f}^{2} \times N_{f}^{2}}$ and $G_{2}=$ $\mathbf{E}\left[\mathcal{C}^{f l} \otimes \mathcal{C}^{f l}\right] \in \mathbb{R}^{N_{f}^{2} \times N_{l}^{2}}$.

The right hand side of (9) converges to a finite value as $k$ goes to infinity if and only if the spectral radius of $G_{1}$ is less than one [37]. In this case, equation (9) tends to

$$
\begin{aligned}
& \operatorname{vec}\left(\Xi_{\infty}^{f}\right)= \\
& \eta^{2} \frac{\pi^{2}}{3}\left(I_{N_{f}} \otimes I_{N_{f}}-G_{1}\right)^{-1}\left(G_{2} \operatorname{vec}\left(I_{N_{l}}\right)+\operatorname{vec}\left(I_{N_{f}}\right)\right) .
\end{aligned}
$$

Notably, such spectral radius determines the convergence speed of followers' state toward the leaders' state in the absence of noise. In what follows, we refer to the spectral radius of $G_{1}$ as $r_{a}$, consistently with the comprehensive analysis of convergence rates in stochastic consensus protocols in Ref. [28].

\subsection{Spectral analysis}

Here, we determine closed-form expressions for $G_{1}$ and $G_{2}$ using a counting argument similar to Refs. [22, 33, 35]. Specifically, we compute $\mathbf{E}[\mathcal{C} \otimes \mathcal{C}]$ and extract such matrices from it based on the decomposition in (5). At each time step, the matrix $\mathcal{C}(k) \otimes \mathcal{C}(k)$ has $N^{4}$ entries $\mathcal{C}_{i j}(k) \mathcal{C}_{s t}(k)$, for $i, j, s, t=1, \ldots, N$ which can take only two values, that is, 0 or $\frac{1}{K^{2}}$. Such entries can be partitioned into four separate cases: (i) $i=s$ and $j=t$, (ii) $i=s$ and $j \neq t$, (iii) $i \neq s$ and $j=t$, and (iv) $i \neq s$ and $j \neq t$. Using a combinatoric argument, the probability to obtain a non-null value is $\frac{K}{N}$ for case (i), $\frac{K(K-1)}{N(N-1)}$ for case (ii), and $\left(\frac{K}{N}\right)^{2}$ for cases (iii) and (iv). We introduce four coefficients, $\alpha_{1}=\frac{1}{K N}, \alpha_{2}=\frac{K-1}{K N(N-1)}$, and $\alpha_{3}=\alpha_{4}=\frac{1}{N^{2}}$ for such entries. The random variable $\mathbf{E}[\mathcal{C} \otimes \mathcal{C}]$ is then computed as: $\mathbf{E}\left[\mathcal{C}_{i j} \mathcal{C}_{s t}\right]=\alpha_{1}$ for case (i), $\mathbf{E}\left[\mathcal{C}_{i j} \mathcal{C}_{s t}\right]=\alpha_{2}$ for case (ii), and $\mathbf{E}\left[\mathcal{C}_{i j} \mathcal{C}_{s t}\right]=\alpha_{3}$ for case (iii) and (iv). For ease of illustration, we introduce the following four $N^{2} \times N^{2}$ matrices:

$$
\begin{aligned}
& M_{1}=\operatorname{vec}\left(I_{N}\right) \operatorname{vec}\left(I_{N}\right)^{\mathrm{T}} \\
& M_{2}=(N-1) M_{1}-N \operatorname{vec}\left(I_{N}\right) \operatorname{vec}\left(R_{N}\right)^{\mathrm{T}}, \\
& M_{3}=\frac{1}{N}\left(1_{N^{2}} 1_{N^{2}}^{\mathrm{T}}-M_{1}-M_{2}\right) M_{1} \\
& M_{4}=\frac{1}{N}\left(1_{N^{2}} 1_{N^{2}}^{\mathrm{T}}-M_{1}-M_{2}\right) M_{2}
\end{aligned}
$$

where $R_{N}=I_{N}-\frac{1}{N} 1_{N} 1_{N}^{\mathrm{T}}$ is an orthogonal projection onto $\operatorname{span}\left(1 \frac{\perp}{N}\right)$. The entries of such matrices take values in $\{0,1\}$, and $\operatorname{vec}\left(M_{1}\right), \operatorname{vec}\left(M_{2}\right), \operatorname{vec}\left(M_{3}\right)$, and $\operatorname{vec}\left(M_{4}\right)$ are linearly independent vectors. These matrices also satisfy the matrix product properties: $\left(M_{1}\right)^{2}=N M_{1}, M_{1} M_{2}=$ $N M_{2}, M_{2} M_{3}=N(N-1) M_{1}, M_{2} M_{4}=N(N-1) M_{2}$, $M_{3} M_{1}=N M_{3}, M_{3} M_{2}=N M_{4},\left(M_{4}\right)^{2}=N(N-1) M_{4}$, $M_{4} M_{3}=N(N-1) M_{3}$, and any other matrix product of the form $M_{i} M_{j}$ with $i, j=1, \ldots, 4$ equals $0_{N^{2} \times N^{2}}$. Using these matrices, $\mathbf{E}[\mathcal{C} \otimes \mathcal{C}]$ is given by

$$
\mathbf{E}[\mathcal{C} \otimes \mathcal{C}]=\alpha_{1} M_{1}+\alpha_{2} M_{2}+\alpha_{3} M_{3}+\alpha_{4} M_{4}
$$

Next, we define two groups of sub-matrices, computed from $M_{i}$ in (11) for $i=1, \ldots, 4$, namely, $M_{i}^{f} \in \mathbb{R}^{N_{f}^{2} \times N_{f}^{2}}$, and $M_{i}^{l} \in \mathbb{R}^{N_{f}^{2} \times N_{l}^{2}}$, for $i=1, \ldots, 4$. The first group is obtained by directly replacing $N$ with $N_{f}$ in (11) and, the second group is defined by

$$
\begin{aligned}
& M_{1}^{l}=\operatorname{vec}\left(I_{N_{f}}\right) \operatorname{vec}\left(I_{N_{l}}\right)^{\mathrm{T}}, \\
& M_{2}^{l}=\left(N_{l}-1\right) M_{1}^{l}-N_{l} \operatorname{vec}\left(I_{N_{f}}\right) \operatorname{vec}\left(R_{N_{l}}\right)^{\mathrm{T}}, \\
& M_{3}^{l}=\frac{1}{N_{f}}\left(1_{N_{f}^{2}} 1_{N_{f}^{2}}^{\mathrm{T}}-M_{1}^{f}-M_{2}^{f}\right) M_{1}^{l}, \\
& M_{4}^{l}=\frac{1}{N_{f}}\left(1_{N_{f}^{2}} 1_{N_{f}^{2}}^{\mathrm{T}}-M_{1}^{f}-M_{2}^{f}\right) M_{2}^{l},
\end{aligned}
$$

where $R_{N_{l}}=I_{N_{l}}-\frac{1}{N_{l}} 1_{N_{l}} 1_{N_{l}}^{\mathrm{T}}$ is an orthogonal projection onto $\operatorname{span}\left(1 \frac{\perp}{N_{l}}\right)$ (Note that in general, $M_{i}^{l}, i=1, \ldots, 4$ are 
not square matrices.). Analytical expressions for $G_{1}$ and $G_{2}$ are then obtained by inspection from $\mathbf{E}[\mathcal{C} \otimes \mathcal{C}]$, that is,

$$
\begin{aligned}
& G_{1}=\alpha_{1} M_{1}^{f}+\alpha_{2} M_{2}^{f}+\alpha_{3} M_{3}^{f}+\alpha_{4} M_{4}^{f}, \\
& G_{2}=\alpha_{1} M_{1}^{l}+\alpha_{2} M_{2}^{l}+\alpha_{3} M_{3}^{l}+\alpha_{4} M_{4}^{l} .
\end{aligned}
$$

It can be verified that $G_{1}$ is a non-symmetric matrix of rank 2. In particular, the eigenvectors of $G_{1}$ corresponding to nontrivial eigenvalues are $\operatorname{vec}\left(1_{N_{f}} 1_{N_{f}}^{\mathrm{T}}\right)+$ $\beta_{1,2} \operatorname{vec}\left(I_{N_{f}}\right)$, where $\beta_{1,2}=\frac{N-K((1-\phi) N+1)}{2 K}(1 \pm \sqrt{\Delta})$ and $\Delta=1+\frac{4 K \phi N(N-K)}{(N-1)(K((1-\phi) N+1)-N)^{2}} \geq 1$. The corresponding eigenvalues are

$$
\lambda_{1,2}=(1-\phi) \frac{1}{K}+\frac{(1-\phi)((1-\phi) N-1)}{(N-1)\left(1+\beta_{1,2}\right)} \frac{K-1}{K} .
$$

By noting that $1-\phi \in(0,1),\left|\frac{(1-\phi)((1-\phi) N-1)}{(N-1)\left(1+\beta_{1,2}\right)}\right| \in(0,1)$, and using the classical convex hulls theorem [36], we have $\left|\lambda_{1,2}\right|<1$. Thus, we can formulate the following proposition:

Proposition 1. The spectral radius of $G_{1}$ in (9) is $r_{a}=$ $\max \left\{\left|\lambda_{1}\right|,\left|\lambda_{2}\right|\right\}$, where $\lambda_{1,2}$ are given by (15). Such spectral radius is less than 1 , so that in the limit $k \rightarrow \infty$ : i) the second moment matrix of the linearized VNM in (3) converges to zero in the absence of noise and the followers reach the leaders' state in the mean square sense; and ii) the second moment matrix remains finite in the presence of noise.

One can also verify that $G_{2}$ is a non-square matrix of rank less or equal to $\min \left\{N_{f}, N_{l}\right\}$. We do not offer further insight into the spectral properties of $G_{2}$, since they do not affect the error second moment matrix, which we seek to characterize, see (9).

In the thermodynamic limit, $N \rightarrow \infty$, we have

$$
r_{a} \simeq \frac{1-\phi}{K}(1+(1-\phi)(K-1)) .
$$

Equation (16) suggests that the proportion of leaders $\phi$ has a remarkable effect on $r_{a}$, whereby increasing $\phi$ produces a decrease in $r_{a}$, which corresponds to a faster dynamics. On the other hand, the number of connected neighbors $K$ has a secondary role; specifically, for $K \gg \frac{1}{1-\phi}, r_{a}$ is approximately independent of $K$. We also comment that for a fixed proportion of leaders, $r_{a}$ is minimized for $K \rightarrow \infty$ and the corresponding minimum is $(1-\phi)^{2}$. On the other hand for a fixed value of $K, r_{a}$ is minimized for $\phi \rightarrow 1$, with a minimum value of 0 , which corresponds to an infinitely fast consensus protocol in the absence of noise.

\section{Steady-state coordination}

\subsection{Polarization}

A well-known measure of the level of coordination for the VNM $[14,18,22]$ is the polarization, which ranges from 0 to 1 as the coordination of the system changes from complete disorder to complete order. Such an order parameter is defined as

$$
\mathrm{Pol}=\lim _{k \rightarrow \infty} \mathbf{E}\left[\frac{1}{N}\left|\sum_{i=1}^{N} e^{\mathrm{I} \theta_{i}(k)}\right|\right] .
$$

For $\eta \ll 1$, the polarization can be written in terms of the steady-state mean square deviation $\delta_{\infty}=\lim _{k \rightarrow \infty} \delta(k)$ following a line of argument similar to Ref. [22]. Toward this aim, (17) is approximated by substituting in $\theta_{i}(k)=\varepsilon_{i}(k)+\theta_{0}$, expanding $\mathrm{e}^{\mathrm{I} \varepsilon_{i}(k)}$ to the second order, and consistently approximating the modulus. Thus, we find

$$
\mathrm{Pol} \simeq 1-\frac{1}{2 N} \delta_{\infty}+\frac{1}{2} \lim _{k \rightarrow \infty} \mathbf{E}\left[\bar{\varepsilon}^{2}(k)\right] .
$$

To compute $\delta_{\infty}$, we left multiply equation $(7)$ by $\operatorname{vec}\left(I_{N}\right)^{\mathrm{T}}$ to obtain

$$
\begin{aligned}
\delta(k) & =\mathbf{E}\left[\|\varepsilon(k)\|^{2}\right] \\
& =\operatorname{vec}\left(I_{N}\right)^{\mathrm{T}} \operatorname{vec}\left(\mathbf{E}\left[\varepsilon(k) \varepsilon(k)^{\mathrm{T}}\right]\right) \\
& =\delta^{f}(k)+\delta^{l}(k) .
\end{aligned}
$$

where $\delta^{f}(k)=\mathbf{E}\left[\left\|\varepsilon^{f}(k)\right\|^{2}\right]$ and $\delta^{l}(k)=\mathbf{E}\left[\left\|\varepsilon^{l}(k)\right\|^{2}\right]$ are the mean square deviations norm for the followers' and the leaders' states, respectively. To calculate $\mathbf{E}\left[\bar{\varepsilon}^{2}(k)\right]$, which corresponds to the second moment of the average disagreement, we note that

$$
\begin{aligned}
\mathbf{E}\left[\bar{\varepsilon}^{2}(k)\right] & =\frac{1}{N^{2}} \mathbf{E}\left[\left(\sum_{i=1}^{N} \varepsilon_{i}(k)\right)^{2}\right] \\
& =\frac{1}{N^{2}} \operatorname{vec}\left(1_{N} 1_{N}^{\mathrm{T}}\right)^{\mathrm{T}} \operatorname{vec}\left(\mathbf{E}\left[\varepsilon(k) \varepsilon(k)^{\mathrm{T}}\right]\right) .
\end{aligned}
$$

By isolating the leaders from the followers, we obtain

$$
\begin{aligned}
\mathbf{E}\left[\bar{\varepsilon}^{2}(k)\right] & =\frac{1}{N^{2}}\left(\operatorname{vec}\left(1_{N_{f}} 1_{N_{f}}^{\mathrm{T}}\right)^{\mathrm{T}} \operatorname{vec}\left(\mathbf{E}\left[\varepsilon^{f}(k) \varepsilon^{f}(k)^{\mathrm{T}}\right]\right)\right. \\
& \left.+\operatorname{vec}\left(1_{N_{l}} 1_{N_{l}}^{\mathrm{T}}\right)^{\mathrm{T}} \operatorname{vec}\left(\mathbf{E}\left[\varepsilon^{l}(k) \varepsilon^{l}(k)^{\mathrm{T}}\right]\right)\right) .
\end{aligned}
$$

The steady-state mean square deviation $\delta_{\infty}^{l}$ for the leaders is computed by applying the operator vec $(\cdot)$ to (8), and left multiplying the result by $\operatorname{vec}\left(I_{N_{l}}\right)^{\mathrm{T}}$, that is,

$$
\delta_{\infty}^{l}=\eta^{2} \frac{\pi^{2}}{3} N_{l} .
$$

The computation of $\delta_{\infty}^{f}$ requires a few additional steps. From (14a), it can be verified that the inverse of the matrix $I_{N_{f}} \otimes I_{N_{f}}-G_{1}$ is

$$
\begin{aligned}
& \left(I_{N_{f}} \otimes I_{N_{f}}-G_{1}\right)^{-1}= \\
& I_{N_{f}} \otimes I_{N_{f}}+\gamma_{1} M_{1}^{f}+\gamma_{2} M_{2}^{f}+\gamma_{3} M_{3}^{f}+\gamma_{4} M_{4}^{f},
\end{aligned}
$$


where

$$
\begin{aligned}
\gamma_{1} & =\frac{\alpha_{1}-N_{f}\left(N_{f}-1\right)\left(\alpha_{1}-\alpha_{2}\right) \alpha_{3}}{\Gamma}, \\
\gamma_{2,3} & =\frac{\alpha_{2,3}}{\Gamma}, \\
\gamma_{4} & =\frac{\alpha_{3}-N_{f}\left(\alpha_{1}-\alpha_{2}\right) \alpha_{3}}{\Gamma},
\end{aligned}
$$

and

$\Gamma=N_{f}^{2}\left(N_{f}-1\right)\left(\alpha_{1}-\alpha_{2}\right) \alpha_{3}-\left(N_{f} \alpha_{1}-1\right)-N_{f}\left(N_{f}-1\right) \alpha_{3}$.

Using the properties of the sub-matrices $M_{i}^{f}$ and $M_{i}^{l}$ for $i=1, \ldots, 4$, we can state the following proposition:

Proposition 2. In the limit $k \rightarrow \infty$, the second moment matrix of the followers' disagreement state in (6a) converges to

$$
\begin{aligned}
\operatorname{vec}\left(\Xi_{\infty}^{f}\right)=\eta^{2} \frac{\pi^{2}}{3}( & \left(N \gamma_{1}+1\right) \operatorname{vec}\left(I_{N_{f}}\right) \\
& \left.+N \gamma_{3}\left(\operatorname{vec}\left(1_{N_{f}} 1_{N_{f}}^{\mathrm{T}}\right)-\operatorname{vec}\left(I_{N_{f}}\right)\right)\right),
\end{aligned}
$$

where $\gamma_{1}$ and $\gamma_{3}$ are given in (24).

The steady-state mean square deviation $\delta_{\infty}^{f}$ for the followers is thus obtained by left-multiplying $(25)$ by $\operatorname{vec}\left(I_{N_{f}}\right)^{\mathrm{T}}$ and using the identities $\operatorname{vec}\left(I_{N_{f}}\right)^{\mathrm{T}} \operatorname{vec}\left(I_{N_{f}}\right)=N_{f}$ and $\operatorname{vec}\left(I_{N_{f}}\right)^{\mathrm{T}} \operatorname{vec}\left(I_{N_{l}}\right)=N_{l}=N-N_{f}$ to find

$$
\delta_{\infty}^{f}=\eta^{2} \frac{\pi^{2}}{3} N_{f}\left(1+N \gamma_{1}\right) .
$$

Finally, we substitute (26) and (22) into (19) to compute the steady state mean square deviation for the entire system

$$
\delta_{\infty}=\eta^{2} \frac{\pi^{2}}{3} N\left(1+N_{f} \gamma_{1}\right) .
$$

Following a similar line of arguments, using equation (8) and Proposition 2, we find the following expression for the steady state second moment of the average disagreement

$$
\lim _{k \rightarrow \infty} \mathbf{E}\left[\bar{\varepsilon}^{2}(k)\right]=\eta^{2} \frac{\pi^{2}}{3} \frac{1}{N}\left(1+\gamma_{1} N_{f}+\gamma_{3}\left(N_{f}^{2}-N_{f}\right)\right) .
$$

Equations (27) and (28) can be replaced in (18) to compute a closed-form expression for the polarization of the VNM model in the presence of leaders. We formulate such result in the following corollary, where, for convenience, we replace $N_{f}=(1-\phi) N$ in $(24 \mathrm{a})$ :

Corollary 1. For $\eta \ll 1$, the polarization (17) of the $V N M$ in the presence of leaders (1) and (2) is

$$
\mathrm{Pol} \simeq 1-\frac{\eta^{2} \frac{\pi^{2}}{6}\left(1-(1-\phi)\left(1-\phi-\frac{1}{N}\right)-\frac{1}{N}\right)}{1-\frac{1-\phi}{K}\left(1+\left(1-\phi-\frac{1}{N}\right) \frac{(K-1+\phi) N-K \phi}{N-1}\right)} .
$$

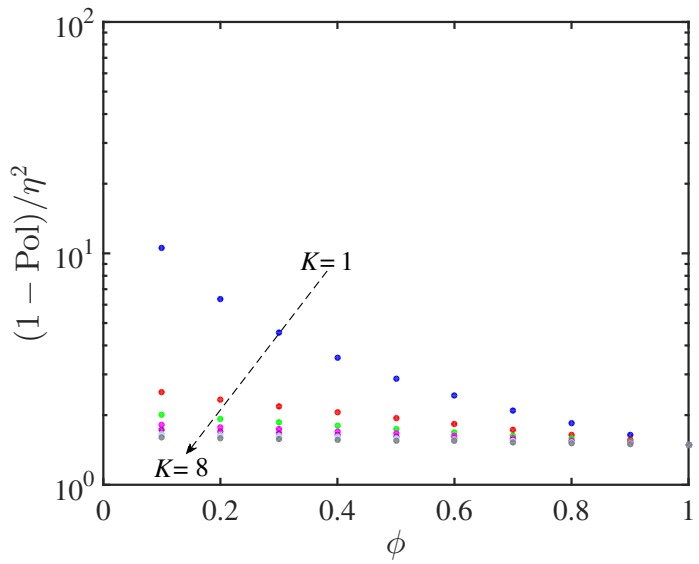

(a)

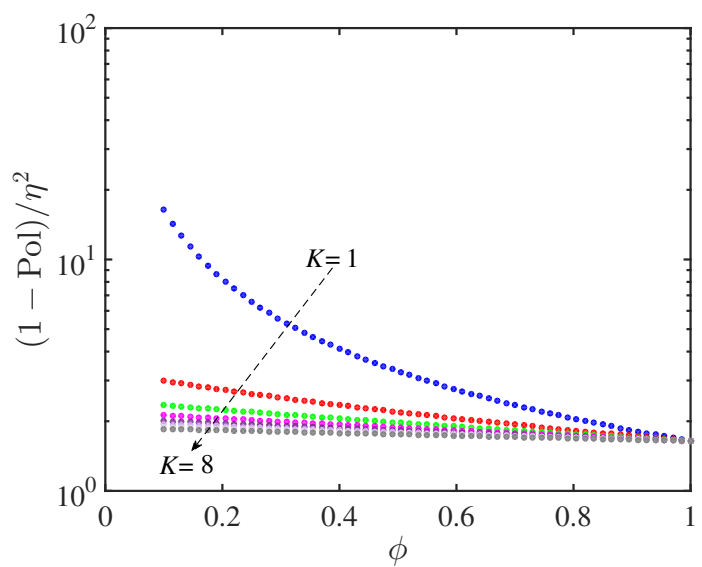

(b)

Figure 1: Plot of $(1-\mathrm{Pol}) / \eta^{2}$ in terms of the number of connected neighbors $K$ from 1 to 8 and the proportion of leaders $\phi$ for $N=10$ (a) and $N=5000$ (b). Note that both $K$ and $\phi$ are discrete variables.

\subsection{Analysis}

The closed-form expression (29) confirms that, for any group size $N$, number of connected neighbors $K$, and proportion of leaders $\phi$, the polarization is a decreasing function of the noise level $\eta$. Figures 1(a) and 1(b) illustrate the effects of the proportion of leaders and the number of connected neighbors on the polarization in terms of $(1-\mathrm{Pol}) / \eta^{2}$, for network of $N=10$ and $N=5000$ nodes. Therein, we notice that the polarization is an increasing function of both $\phi$ and $K$. The dependence on $\phi$ is more evident for smaller values of $K$, whereby the curves in Figure 1 tend to plateau as $K$ increases. In addition, we comment that the dependence on $K$ is highly nonlinear, whereby increasing $K$ from 1 to 2 produces significant changes in the polarization which are not observed for unit increases at larger values of $K$.

With reference to the larger network considered in Figure 1 (b), for $N \gg 1$ and $N_{l} \gg 1$, the polarization can be well approximated as $\mathrm{Pol} \simeq 1-\eta^{2} \frac{\pi^{2}}{6} \frac{K}{K-1+\phi}$. In this case, a crude estimate of the critical noise level at which the transition from order to disorder is observed is $\eta_{c}=$ 
$\frac{\sqrt{6}}{\pi} \sqrt{\frac{K-1+\phi}{K}}$, which is computed by setting Pol $=0$ in (29), similar to [22]. Notably, for $K \geq 2$, the proportion of leaders $\phi$ plays a secondary role on $\eta_{c}$, which is controlled by the number of connected neighbors $K$. Thus, our linear analysis suggests that the presence of leaders only weakly influences the state-transition of the VNM, which is regulated by $K$.

We also note that for $K=1$ and $N \gg 1$, the analytical expression for the polarization reduces to $\mathrm{Pol} \simeq 1-\eta^{2} \frac{\pi^{2}}{6} \frac{1}{\phi}$. In this case, the critical noise level is $\eta_{c}=\frac{\sqrt{6 \phi}}{\pi}$, which suggests that coordination of the system is possible only for minimal noise levels, due to the presence of group leaders.

On the other hand, for $K=N$, which corresponds to all-to-all coupling, we have

$$
\mathrm{Pol} \simeq 1-\eta^{2} \frac{\pi^{2}}{6}\left(1-\frac{1}{(2-\phi) N}\right) .
$$

In the thermodynamic limit, ultimately, the above expression reduces to $\mathrm{Pol} \simeq 1-\eta^{2} \frac{\pi^{2}}{6}$. Thus, we have $\eta_{c}=\frac{\sqrt{6}}{\pi}$ and coordination is possible for higher levels of noise.

Finally, we comment that, independently of the number of connected neighbors $K$ and the group size $N$, the polarization for a large proportion of leaders, that is, $\phi \rightarrow 1$, reduces to $\mathrm{Pol}=1-\eta^{2} \frac{\pi^{2}}{6}\left(1-\frac{1}{N}\right)$. In this case, all the agents are leaders that maintain their state under the effect of noise.

\subsection{Validation against nonlinear situation results}

We compare analytical results from the closed-form expression in (29) to numerical simulations of the original model in (2). We simulate the order parameter with network sizes of $N=10$ nodes and $N=5000$ nodes, similar to Figure 1. In both cases, the model is initialized with $\theta_{i}(0)=0$, for $i=1, \ldots, N$. For the case of $N=5000$ nodes, the model is executed for 300 time steps and the last 100 time steps are averaged to compute the polarization. For the small network size of $N=10$ nodes, the simulation is instead executed for 10000 time steps, and the last 8000 time steps are averaged to compute the polarization.

Table 1: Comparison between analytical $\eta^{\text {an }}$ and numerical $\eta^{\text {num }}$ results for $N=10$ and $\phi=0.1$. Subscripts refer to the value of the polarization.

\begin{tabular}{c||cccccccccc}
\hline \hline$K$ & 1 & 2 & 3 & 4 & 5 & 6 & 7 & 8 & 9 & 10 \\
\hline$\eta_{0.90}^{\text {num }}$ & 0.10 & 0.20 & 0.22 & 0.23 & 0.23 & 0.24 & 0.24 & 0.24 & 0.25 & 0.25 \\
$\eta_{0.90}^{\text {an }}$ & 0.10 & 0.20 & 0.22 & 0.23 & 0.24 & 0.24 & 0.25 & 0.25 & 0.25 & 0.25 \\
\hline$\eta_{0.75}^{\text {num }}$ & 0.17 & 0.31 & 0.35 & 0.37 & 0.38 & 0.38 & 0.39 & 0.39 & 0.40 & 0.40 \\
$\eta_{0.75}^{\text {an }}$ & 0.15 & 0.31 & 0.35 & 0.37 & 0.38 & 0.39 & 0.39 & 0.40 & 0.40 & 0.40 \\
\hline$\eta_{0.50}^{\text {num }}$ & 0.31 & 0.44 & 0.49 & 0.52 & 0.54 & 0.55 & 0.56 & 0.57 & 0.57 & 0.58 \\
$\eta_{0.50}^{\text {an }}$ & 0.22 & 0.44 & 0.50 & 0.52 & 0.54 & 0.55 & 0.55 & 0.56 & 0.56 & 0.57 \\
\hline
\end{tabular}

Figures 2 and 3 compare numerical simulations to analytical results a function of the noise level $\eta$ for three values of the number of $(K=2,5$, and 8$)$. For the large network size considered in Figure 3, the order parameter

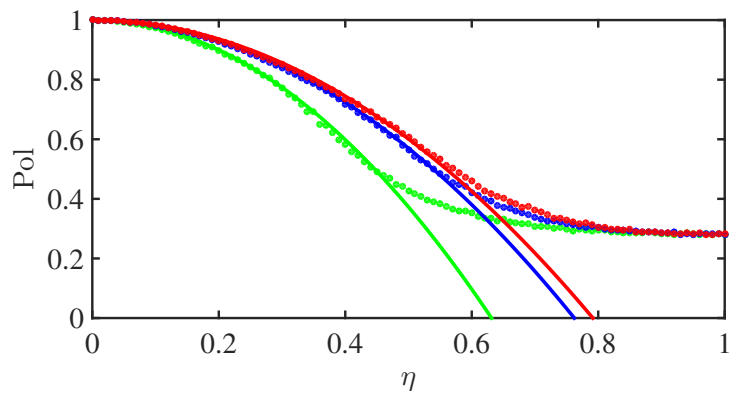

Figure 2: Polarization as a function of the noise level for different values of $K(K=2$ in green, $K=5$ in blue, $K=8$ in red) with $N=10$ and $\phi=0.1$. Solid lines are analytical predictions from (29) and dots are numerical simulations. Note that $\phi=0.1$ corresponds to one leader and 9 followers.

Table 2: Comparison between analytical $\eta^{\text {an }}$ and numerical $\eta^{\text {num }}$ results for $N=5000$ and $\phi=0.001$. Subscripts refer to the value of the polarization.

\begin{tabular}{c||cccccccccc}
\hline \hline$K$ & 1 & 2 & 3 & 4 & 5 & 6 & 7 & 8 & 9 & 10 \\
\hline$\eta_{0.90}^{\text {num }}$ & 0.01 & 0.17 & 0.20 & 0.21 & 0.22 & 0.22 & 0.23 & 0.23 & 0.23 & 0.23 \\
$\eta_{0.90}^{\text {nan }}$ & 0.01 & 0.17 & 0.20 & 0.21 & 0.22 & 0.22 & 0.23 & 0.23 & 0.23 & 0.23 \\
\hline$\eta_{0.75}^{\text {num }}$ & 0.02 & 0.28 & 0.33 & 0.35 & 0.35 & 0.36 & 0.37 & 0.38 & 0.38 & 0.38 \\
$\eta_{0.75}^{\text {an }}$ & 0.02 & 0.28 & 0.32 & 0.34 & 0.35 & 0.35 & 0.36 & 0.36 & 0.37 & 0.37 \\
\hline$\eta_{0.50}^{\text {num }}$ & 0.03 & 0.35 & 0.45 & 0.49 & 0.52 & 0.53 & 0.54 & 0.55 & 0.56 & 0.56 \\
$\eta_{0.50}^{\text {an }}$ & 0.02 & 0.39 & 0.45 & 0.48 & 0.49 & 0.50 & 0.51 & 0.51 & 0.52 & 0.53 \\
\hline$\eta_{c}^{\text {num }}$ & 0.08 & 0.39 & 0.51 & 0.57 & 0.61 & 0.64 & 0.67 & 0.68 & 0.70 & 0.71 \\
$\eta_{c}^{\text {an }}$ & 0.08 & 0.55 & 0.64 & 0.67 & 0.70 & 0.71 & 0.72 & 0.73 & 0.74 & 0.74 \\
\hline
\end{tabular}

in the VNM containing leaders exhibits a phase transition from order to complete disorder. A similar behavior is observed in the VNM without leaders $[14,18,22]$ and in self-propelled particle models $[15,16,19]$. Not only does the closed-form expression capture the general trend of the polarization as a function of the independent variables, but it is also able to closely predict numerical findings for $\eta \leq 0.3$ for the small network size and for $\eta \leq 0.35$ for the large network size. This observation is further supported by data in Tables 1 and 2, which displays the values of $\eta$ for which $\mathrm{Pol}=0.9, \mathrm{Pol}=0.75$ and $\mathrm{Pol}=0.5$, computed both numerically and analytically. In the opposite case of large noise intensities, the polarization can be estimated by hypothesizing that the orientation of the followers are i.i.d. random variables with common uniform random variable taking values in $[-\pi, \pi]$ and computing the expectation in (18). Such computation would yield 0.28 for Figure 2 and zero for Figure 3.

The comparison between analytical results and numerical simulations presented in Tables 1 and 2 demonstrates that numerical and analytical findings are almost identical for the prediction of the noise level which leads to a drop of the polarization to 0.90 . With respect to the cases $\mathrm{Pol}=0.75$ and $\mathrm{Pol}=0.50$, except for $K=1$, the results obtained for both methods are almost identical for the small network size and differ for only $3 \%$ for the large group size. 


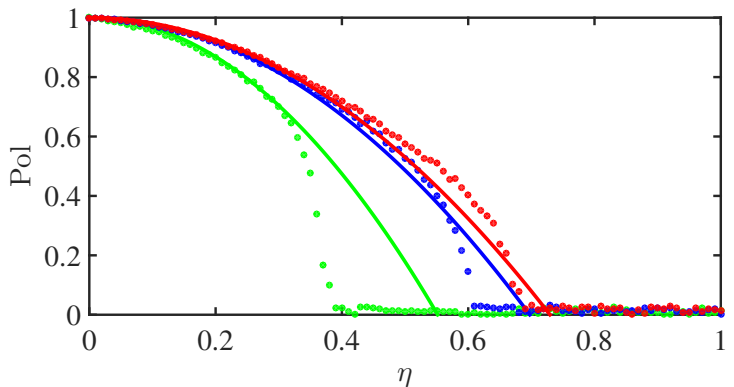

Figure 3: Polarization as a function of the noise level for different values of $K(K=2$ in green, $K=5$ in blue, $K=8$ in red) with $N=5000$ and $\phi=0.001$. Solid lines are analytical predictions from (29) and dots are numerical simulations. Note that $\phi=0.001$ corresponds to 5 leaders and 4995 followers.

\section{Conclusions}

In this work, the linear stochastic behavior of the vectorial network model in the presence of leaders has been investigated. We have considered the system dynamics in the vicinity of a synchronized state, in which the orientations of the leaders and followers are proximal. By analyzing such linear stochastic system, we have established a toolbox of closed-form analytical findings that enable dissecting the effects of the group size, the number of connected neighbors, the noise intensity, and the proportion of leaders on the system coordination. Our findings have been validated through numerical simulations on small and large networks.

Future research should seek to investigate the role of leaders with different orientations that could vary in time, explore more refined models for the noise, expand on the theoretical framework to predict the group coordination for larger noise intensities.

\section{Acknowledgment}

The authors would like to thank J. Laut for the careful review of the manuscript.

[1] J. Krause, G. D. Ruxton, Living in groups, Oxford University Press, 2002.

[2] D. J. Sumpter, The principles of collective animal behaviour, Philosophical Transactions of the Royal Society B: Biological Sciences 361 (2006) 5-22.

[3] N. Smelser, Theory of collective behavior, Routledge, 2011.

[4] I. D. Couzin, J. Krause, N. R. Franks, S. A. Levin, Effective leadership and decision-making in animal groups on the move, Nature 433 (2005) 513-516.

[5] A. Cavagna, A. Cimarelli, I. Giardina, G. Parisi, R. Santagati, F. Stefanini, M. Viale, Scale-free correlations in starling flocks, Proceedings of the National Academy of Sciences 107 (2010) 11865-11870.

[6] J. Kennedy, R. Eberhart, et al., Particle swarm optimization, in: Proceedings of the IEEE International Conference on Neural Networks, volume 4, Perth, Australia, pp. 1942-1948.

[7] A. J. Ijspeert, Central pattern generators for locomotion control in animals and robots: a review, Neural Networks 21 (2008) 642-653.

[8] W. Wang, J.-J. Slotine, Contraction analysis of time-delayed communications and group cooperation, IEEE, Transactions on Automatic Control 51 (2006) 712-717.
[9] M. Dorigo, G. Di Caro, L. M. Gambardella, Ant algorithms for discrete optimization, Artificial Life 5 (1999) 137-172.

[10] N. Abaid, M. Porfiri, Fish in a ring: spatiotemporal pattern formation in one-dimensional animal groups, Journal of the Royal Society Interface 7 (2010) 1441-1453.

[11] J. Dyer, A. Johansson, D. Helbing, I. Couzin, J. Krause, Leadership, consensus decision making and collective behaviour in humans, Philosophical Transactions of the Royal Society B: Biological Sciences 364 (2009) 781-789.

[12] Y. Y. Liu, J. J. Slotine, A. L. Barabasi, Controllability of complex networks, Nature 473 (2011) 167-173.

[13] R. Grigoriev, M. Cross, H. Schuste, Pinning control of spatiotemporal chaos, Physical Review Letters 79 (1997) 27952798.

[14] M. Aldana, V. Dossetti, C. Huepe, V. M. Kenkre, H. Larralde, Phase transitions in systems of self-propelled agents and related network models, Physical Review Letters 98 (2007) 95702.

[15] T. Vicsek, A. Czirók, E. Ben-Jacob, I. Cohen, O. Shochet, Novel Type of Phase Transition in a System of Self-Driven Particles, Physical Review Letters 75 (1995) 1226-1229.

[16] A. Czirók, T. Vicsek, Collective behavior of interacting selfpropelled particles, Physica A: Statistical Mechanics and its Applications 281 (2000) 17-29.

[17] T. Vicsek, A. Zafeiris, Collective motion, Physics Reports 517 (2012) 3-4.

[18] J. A. Pimentel, M. Aldana, C. Huepe, H. Larralde, Intrinsic and extrinsic noise effects on phase transitions of network models with applications to swarming systems, Physical Review E 77 (2008) 061138.

[19] H. Chaté, F. Ginelli, G. Grégoire, F. Raynaud, Collective motion of self-propelled particles interacting without cohesion, Physical Review E 77 (2008) 046113.

[20] Y. L. Chou, R. Wolfe, T. Ihle, Kinetic theory for systems of self-propelled particles with metric-free interactions, Physical Review E 86 (2012) 021120.

[21] M. Ballerini, N. Cabibbo, R. Candelier, A. Cavagna, E. Cisbani, I. Giardina, V. Lecomte, A. Orlandi, G. Parisi, A. Procaccini, et al., Interaction ruling animal collective behavior depends on topological rather than metric distance: Evidence from a field study, Proceedings of the National Academy of Sciences 105 (2008) 1232-1237.

[22] M. Porfiri, Linear analysis of the vectorial network model, Express Briefs, IEEE Transactions on Circuits and Systems II 61 (2014) 44-48.

[23] Y. Hatano, M. Mesbahi, Agreement over random networks, IEEE Transactions on Automatic Control 50 (2005) 1867-1872.

[24] M. Huang, J. H. Manton, Stochastic consensus seeking with noisy and directed inter-agent communication: fixed and randomly varying topologies, IEEE Transactions on Automatic Control 55 (2010) 235-241.

[25] M. Porfiri, D. J. Stilwell, Consensus seeking over random weighted directed graphs, IEEE Transactions on Automatic Control 52 (2007) 1767-1773.

[26] A. Tahbaz-Salehi, A. Jadbabaie, A necessary and sufficient condition for consensus over random networks, IEEE Transactions on Automatic Control 53 (2008) 791-795.

[27] S. S. Pereira, A. Pages-Zamora, Mean square convergence of consensus algorithms in random WSNs, IEEE Transactions on Signal Processing 58 (2010) 2866-2874.

[28] J. Zhou, Q. Wang, Convergence speed in distributed consensus over dynamically switching random networks, Automatica 45 (2009) 1455-1461.

[29] S. Kar, J. M. F. Moura, Sensor networks with random links: Topology design for distributed consensus, IEEE Transactions on Signal Processing 56 (2008) 3315-3326.

[30] S. Patterson, B. Bamieh, A. El Abbadi, Convergence rates of distributed average consensus with stochastic link failures, IEEE Transactions on Automatic Control 55 (2010) 880-892.

[31] C. W. Wu, Synchronization and convergence of linear dynamics in random directed networks, IEEE Transactions on Automatic Control 51 (2006) 1207-1210. 
[32] Y. Cao, W. Yu, W. Ren, G. Chen, An overview of recent progress in the study of distributed multi-agent coordination, IEEE Transactions on Industrial Informatics 9 (2013) 427-438.

[33] N. Abaid, M. Porfiri, Consensus over numerosity-constrained random networks, IEEE Transactions on Automatic Control 56 (2011) 649-654.

[34] N. Abaid, I. Igel, M. Porfiri, On the consensus protocol of conspecific agents, Linear Algebra and its Applications 437 (2012) 221-235.

[35] N. Abaid, M. Porfiri, Leader-follower consensus over numerosity-constrained random networks, Automatica 48 (2012) 1845-1851.

[36] D. S. Bernstein, Matrix mathematics, Princeton University Press, Princeton, NJ, 2009.

[37] R. A. Horn, C. R. Johnson, Matrix analysis, Cambridge University Press, Cambridge, UK, 1985. 